\title{
«La langue est une marchandise ». Regards sur la diffusion du français auprès de publics spécialisés du Moyen Âge au XX ${ }^{\mathrm{e}}$ siècle
}

gisele.kahn@gmail.com

"Language is a commodity". A look at the diffusion of French to specialized publics from the Middle Ages to the 20th century

Gisèle Kahn

\section{OpenEdition}

\section{Édition électronique}

URL : https://journals.openedition.org/dhfles/8125

DOI : $10.4000 /$ dhfles. 8125

ISSN : 2221-4038

\section{Éditeur}

Société Internationale pour l'Histoire du Français Langue Étrangère ou Seconde

\section{Édition imprimée}

Date de publication : 1 décembre 2021

ISSN : 0992-7654

\section{Référence électronique}

Gisèle Kahn, " "La langue est une marchandise ». Regards sur la diffusion du français auprès de publics spécialisés du Moyen Âge au XXe siècle », Documents pour l'histoire du français langue étrangère ou seconde [En ligne], 66-67 | 2021, mis en ligne le 11 février 2022, consulté le 26 mars 2023. URL http://journals.openedition.org/dhfles/8125; DOI : https://doi.org/10.4000/dhfles.8125

Ce document a été généré automatiquement le 26 mars 2023.

Tous droits réservés 


\title{
« La langue est une marchandise ». Regards sur la diffusion du français auprès de publics spécialisés du Moyen Âge au XX ${ }^{\mathrm{e}}$ siècle
}

\author{
gisele.kahn@gmail.com \\ "Language is a commodity". A look at the diffusion of French to specialized \\ publics from the Middle Ages to the 20th century
}

\section{Gisèle Kahn}

1 Au fil des siècles et dans différentes régions du monde, apparaît épisodiquement une exigence accrue de formation de professionnels de différents domaines, généralement de haut niveau. Les motifs, économiques, politiques, scientifiques ou de concurrence entre les pays sont divers. Ils émanent de décisions prises par les autorités locales tout comme de pressions étrangères. L'étude des langues, le français parmi d'autres, constitue souvent un passage obligé des dispositifs mis en place. L'histoire de l'enseignement du français ou en français à des publics spécialisés dans différents lieux a déjà fait l'objet de travaux plus ou moins approfondis.

2 Pour ce qui est des périodes anciennes, on peut les découvrir notamment dans les publications de la SIHFLES, de façon détaillée ou juste par allusion. On en verra quelques-uns, qui portent sur les professions les plus étudiées : marchands, médecins, scientifiques, militaires, interprètes, le tout dans des zones géographiques très éparpillées, en Europe, Asie, Proche-Orient, et selon une chronologie désordonnée, du Moyen Âge aux premières années du $\mathrm{XX}^{\mathrm{e}}$ siècle. Les différentes situations repérées montrent de nombreuses similitudes, entre elles d'une part, mais également avec des situations récentes, en particulier dans les objectifs, les formes d'implantation et les modes de financement.

On abordera dans une seconde partie ce qui concerne les années 1960 et suivantes qui ont vu un intérêt renouvelé pour les questions portant sur l'enseignement du français à 
vocation professionnelle, avec souvent des préoccupations proches de celles observées dans des périodes plus anciennes. L'accent sera mis pour l'essentiel sur la dimension géopolitique et institutionnelle des situations présentées, les aspects pédagogiques, certes non négligeables et parfois bien documentés, étant tout au plus évoqués dans cette contribution.

\section{Quelques jalons anciens}

\section{Les marchands, les voyageurs}

4 L'Europe comporte un important réseau de villes marchandes. Acheteurs et négociants circulent. À leur intention, on publie des dictionnaires multilingues et des manuels inspirés notamment de Berlaimont ( $\dagger$ 1531): de deux langues au départ, français et flamand, on passe à quatre, puis cinq, puis huit. En voici un exemple, parmi d'autres, consultable en ligne, publié en 1613 à Flessingue: Colloquia et dictionario cum octo linguarum [...] Colloques ou Dialogues, avec un Dictionaire en huit languages [...] redigez \& augmentez de quatre dialogues, tres profitables \& utils, tant au fait de marchandise, qu'aux voyages \& autres traffiques [...].

Des versions en huit langues sont imprimées dans toute l'Europe jusqu'au milieu du XVIII siècle: plus de 150 éditions répertoriées. À la fois vade-mecum faciles à transporter et à consulter pour voyageurs autodidactes et manuels (Colombo Timelli 1992), ces ouvrages proposent des conversations usuelles, des transactions commerciales et des discussions entre négociants dans les diverses langues: on marchande à n'en plus finir le prix d'une pièce de drap sur un marché ; quand il s'agit de payer, rien ne va plus, les monnaies proposées présentent toutes un défaut: leur poids est mauvais, elles sont rognées, démonétisées, fausses... (Aubert 1993 ; CharletMesdjian \& Charlet 2011). On y trouve également des modèles de lettres, pour demander un paiement à un débiteur, pour réclamer une dette, et quelques exemples de contrats, quittances et lettres de change.

6 La publication de dictionnaires et manuels polyglottes destinés aux négociants se poursuit aux XIX et $\mathrm{XX}^{\mathrm{e}}$ siècles, par exemple chez l'éditeur Hoepli à Milan (Gohard Radenkovic 1998; Merger 2009). La correspondance commerciale occupe une place de plus en plus importante dans certains manuels. Des filières techniques destinées aux activités marchandes sont créées dans divers pays et les ouvrages ou parties d'ouvrages utiles suivent. Le répertoire italien, par exemple, en signale un bon nombre, dès le milieu du XVIII ${ }^{\mathrm{e}}$ siècle (Minerva \& Pellandra 1997).

\section{Des disciplines enseignées en français. Un exemple parmi d'autres : l'université de Vilnius}

Un grand nombre de savants étrangers sont venus enseigner à l'Université de Vilnius après le départ des jésuites. Dès l'année 1815, conformément à la tradition académique, toutes les disciplines étaient enseignées en latin. Pourtant certaines matières étaient données en français : par exemple, c'est en français que l'Italien A. Capelli exposait le droit, l'Anglais J. Saunders l'histoire et la philosophie de l'art. Le français était aussi la langue de certains documents officiels. Tous les professeurs de l'université parlaient français. (Cepukieniene 1998 : 134) 
7 Cette citation résume une situation souvent évoquée pour d'autres pays européens. L'université de Vilnius est l'une des plus anciennes d'Europe, fondée en 1578 par transformation de l'ancien collège jésuite lui-même créé en 1570. Jusqu'à la suppression de la Compagnie en 1773, l'université reste dirigée par les jésuites, en 1781 elle devient un établissement laïc. Que la tradition jésuite de l'enseignement du latin et en latin se poursuive aux quatre coins de la planète importe peu ici. Le rôle éducatif joué par la Compagnie, et ce dans toutes les disciplines, est avéré. C'était, c'est encore sa marque de fabrique. Ce qui fait sens dans l'exemple de Vilnius, c'est l'évocation de l'usage de la langue française dans une bonne partie de l'Europe savante et cultivée tout au long des $\mathrm{XVIII}^{\mathrm{e}}$ et $\mathrm{XIX}^{\mathrm{e}}$ siècles. On pense à l'ouvrage de Marc Fumaroli et à son aréopage de locuteurs francophones de toutes origines et aux nombreux réseaux qui ont favorisé l'expansion du français; aux travaux de Lucien Bély (2017: 157-177) sur l'usage diplomatique de la langue française.

\section{La formation comme vecteur du français hors de France}

\section{Au Japon, les militaires}

Obstinément fermé aux influences étrangères occidentales, du XVII ${ }^{e}$ au milieu du XIX siècle, le Japon fait l'objet d'attaques diplomatiques et militaires incessantes. Vers les années 1840, d'aucuns, parmi les responsables japonais soucieux de préserver l'intégrité du pays, estiment urgent d'étudier les sciences occidentales ayant des applications militaires: artillerie, tactique, géographie, fortification, chimie... On s'interroge: "quels livres hollandais fallait-il choisir parmi ceux qui traitaient de la poudre la plus puissante en vue d'une utilisation dans les nouvelles armes à feu " (Tanaka 1983 : 21). Jusqu'à 1854, date à laquelle les États-Unis parviennent à imposer une certaine ouverture au pays, les seuls étrangers autorisés à résider et à exercer des activités commerciales sont des Hollandais de la Compagnie néerlandaise des Indes orientales, qui n'ont pas le droit de quitter l'île de Déjima face à Nagasaki sur laquelle ils sont installés, avec à leurs côtés une cohorte d'interprètes. Après quelques tentatives sporadiques, l'étude du français au Japon commence, de façon anecdotique semble-t-il, avec la commande et l'arrivée d'un ouvrage de chimie du savant suédois Berzelius rédigé contre toute attente en français. Et c'est l'histoire de Murakami Eishun, médecin et passionné de chimie, qui se met tout seul et avec opiniâtreté à l'apprentissage du français à l'aide de quelques outils disponibles confiés pour l'occasion, un dictionnaire français-néerlandais de Pierre Marin, une grammaire de Lhomond rédigée en néerlandais (Tanaka op. cit.).

Une dizaine d'années après, l'ouverture du pays aidant et les traités signés, les pays occidentaux entrent en action et en concurrence. La France à sa façon participe à la modernisation du Japon: envoi d'une mission militaire pour former les troupes du Shogun à l'européenne, construction de l'arsenal de Yokosuka, création de l'école franco-japonaise de Yokohama où doivent être formés des personnels aptes à comprendre le français, entre autres les officiers de la nouvelle armée japonaise, formation de juristes parallèlement à la traduction en japonais des codes napoléoniens (Graziani 2012 : 171-173). On trouve dans l'ouvrage de Tanaka (cf. supra) les détails des recrutements rendus nécessaires par ces implantations et quelques données concernant certains des programmes de formation. 
10 Par ailleurs, selon une formule expérimentée ailleurs ( $c f$. infra), le gouvernement japonais envoie un contingent d'une soixantaine de jeunes étudiants en France, boursiers pour la plupart, capables de devenir ensuite les cadres de leur pays. Leurs spécialités : art militaire (les plus nombreux), droit, mécanique, industrie minière, architecture, agriculture et chimie, économie politique, comptabilité, etc. On peut suivre la carrière de certains d'entre eux à leur retour au Japon (Tanaka, op. cit.).

11 En fait, la présence française, fortement appuyée par les autorités diplomatiques, subit la concurrence, militaire, scientifique, des autres puissances agissantes dans cette région du monde. Après la défaite de la France contre la Prusse en 1870-1871, elle ira s'affaiblissant, là comme ailleurs (Graziani, op. cit.).

\section{L'université Saint-Joseph de Beyrouth}

12 La mise en place de l'université Saint-Joseph de Beyrouth est largement documentée. Fondée en 1875 par les pères jésuites, elle est le premier établissement d'enseignement universitaire catholique et francophone de la région. Son implantation est le résultat d'une convergence d'intérêts entre le Vatican, les jésuites, la hiérarchie maronite et la France, intérêts communautaires, religieux, politiques et régionaux, la France étant particulièrement soucieuse d'affermir son influence économique (Herzstein 2010). Sont créés successivement la faculté de philosophie et de théologie en 1881, l'école française de médecine en 1883, une école de droit et une école d'ingénieurs en 1913, un département des sciences administratives et politiques en 1920, etc.

13 La création de l'école de médecine, considérée par beaucoup comme la plus emblématique, vise à former un corps médical devant exercer dans les villes et les campagnes de la région confrontées à une réelle pénurie de praticiens (Herzstein 2009). Mais elle a également pour but de concurrencer l'école de médecine fondée peu de temps auparavant par des missionnaires américains et soupçonnée de servir les intérêts de l'influence anglaise (Riffier 2001) : «En 1898, Paul Cambon, ambassadeur de France à Constantinople, évoquera en ces termes les raisons qui ont présidé à la création de cet établissement » :

La pensée qui a présidé à la fondation de cette faculté est due à Gambetta et au Cardinal [sic] Lavigerie. Leur idée était de créer en Syrie une grande école française où les jeunes gens du pays viendraient s'instruire dans les sciences médicales, se perfectionner dans l'étude de notre langue, pour se répandre ensuite dans tout l'Orient comme autant d'amis de notre influence et de notre civilisation. La pensée première des fondateurs marque donc bien le but de l'institution : c'est une pensée politique et une institution de propagande. (Riffier op. cit. : 87)

\section{L'université l'Aurore de Shanghai}

Sur fond de concurrence entre les langues, notamment l'anglais, l'université l'Aurore est fondée par les jésuites français en 1903. Il s'agit, comme ailleurs, de former par le biais de l'éducation une élite favorable à la France afin de rivaliser avec les autres nations occidentales. Après diverses péripéties, liées en particulier à la place des différentes langues, l'Aurore comporte un niveau préparatoire qui prépare au baccalauréat, avec un enseignement progressif du français, et un niveau supérieur divisé en deux départements, lettres-droit, sciences-médecine. Les différentes disciplines sont enseignées en français, avec un appui et une aide financière conséquents des autorités françaises. L'Aurore doit une bonne partie de sa notoriété à 
sa faculté de médecine, accolée à l'hôpital Sainte-Marie fondé par les jésuites en 1907, partie de l'ensemble du dispositif de santé à l'occidentale institué par les différentes communautés installées à Shanghai. La sélection des étudiants est stricte, ils doivent avoir un niveau suffisamment élevé pour pouvoir suivre des cours de médecine en français. L'organisation et le contenu des cours sont similaires à ceux proposés en France, ce qui permet des équivalences avec les diplômes français. Le corps professoral, français pour l'essentiel au début, mixte progressivement, est payé par la France (Glaise 2005 ; Brizay 2013).

\section{Demande et offre de formation ou la stratégie des boursiers scientifiques}

\section{Des chirurgiens pour l'Espagne}

15 Tout au long du XVIII ${ }^{e}$ siècle, en Espagne comme ailleurs en Europe, on assiste à une circulation importante de professionnels français. Les échanges entre savants et praticiens des deux pays sont monnaie courante. Les besoins en professionnels qualifiés se font sentir dans des domaines jugés stratégiques, et outre une importante politique de traduction de textes scientifiques et de création de bibliothèques spécialisées, de nombreuses académies, militaires, scientifiques, médicales, sont créées dans différentes villes. La présence et la collaboration de spécialistes français y est importante et leur action est complétée par des voyages et des séjours d'études de leurs homologues espagnols en France. On doit à un médecin catalan formé à Montpellier, Pedro Virgili, la création des Reales Colegios de Cirugia de Cadix et de Barcelone. Il inaugure en Espagne la pratique visant à envoyer un certain nombre de jeunes pensionnés du roi pour compléter leurs études en chirurgie pendant plusieurs années à l'étranger, à Paris entre autres, et au plus haut niveau. Leur formation est organisée et suivie de près de manière conjointe par les deux parties. Le niveau de langue de départ ne semble pas compter dans le choix des candidats, mais parmi les dépenses nécessaires à leur séjour figurent celles destinées à payer des maîtres de langue (Roig 1996).

\section{Des scientifiques pour l'Égypte}

$16 \mathrm{Au}$ retour de l'expédition d'Égypte de Bonaparte à laquelle il a participé en tant qu'ingénieur-géographe, Edme-François Jomard, qui a gardé de nombreuses relations avec les personnalités égyptiennes, s'efforce de convaincre le pacha Méhémet Ali de faire venir en France de jeunes Égyptiens pour les former dans les divers métiers utiles à la modernisation de leur pays (Ibrahim 2007 ; Chadefaud 2012). Après de longues et acrobatiques négociations est créée à Paris en 1826 l'École franco-égyptienne qui va recevoir pendant quelques années plusieurs dizaines d'étudiants devant se former dans les spécialités les plus diverses, les frais d'études étant assumés par l'Égypte. Solidement encadrés, ceux-ci reçoivent d'abord un enseignement de français et de mathématiques pendant un an, les évaluations sont régulières, le suivi individualisé, leur niveau contrôlé par un examen écrit. Ils sont ensuite répartis dans des établissements correspondant aux spécialités retenues pour chacun, à Paris ou en province: administration civile et militaire, marine, artillerie, génie militaire, hydraulique, mines, chimie, médecine, agriculture, traduction..., une quinzaine de domaines au total. Les promotions se succèdent et en 1832, ils sont quatre-vingts en 
formation. L'expérience dure une dizaine d'années. Les aléas politiques et diplomatiques finissent par avoir raison de l'entreprise et en 1835 les étudiants égyptiens sont rappelés dans leur pays.

\section{Drogmans, truchements, traducteurs ou interprètes}

\section{Au Moyen Âge, des « fixeurs "}

De nos jours, on les appellerait des «fixeurs » (Stahuljak 2018). Ce sont généralement des personnes du cru, ayant acquis les langues on ne sait comment, qui servent d'interprètes mais aussi de guides, de médiateurs, d'organisateurs de contacts avec les interlocuteurs locaux. Au Moyen Âge, ils interviennent comme interprètes des moines prêcheurs qui vont porter la bonne parole au Proche et au Moyen-Orient, jusque dans les steppes eurasiatiques. Ils servent aussi de guides, truchements, voire protecteurs des pèlerins qui se rendent en Terre sainte. Ils sont indispensables, mais la question de leur fiabilité et de leur fidélité est posée. Les missionnaires donnent souvent une image négative de leurs "fixeurs ", plus enclins à défendre leurs propres intérêts que ceux de leurs "clients", n'ayant de surcroît ni la formation théologique ni le vocabulaire appropriés pour ce qu'on attend d'eux. Échaudés par les déboires subis, les missionnaires finissent par décider d'apprendre eux-mêmes les langues des contrées traversées ou visées (Balivet 1997 ; Delacroix-Besnier 2012 ; Tolan 2009). D'une certaine façon, les frères prêcheurs peuvent être considérés comme les précurseurs de l'apprentissage organisé des langues à des fins professionnelles, et ce dès le milieu du XIII ${ }^{e}$ siècle.

Certains récits de pèlerins livrent parfois l'état détaillé des dépenses non incluses dans les contrats de départ: sauf-conduits ou droits de passage imprévus, coûts supplémentaires pour s'assurer la bienveillance des passeurs, des aubergistes, dons pour se protéger contre les mauvaises rencontres, sans oublier les vols commis par certains drogmans de mèche avec les chameliers... La langue joue là un rôle central, elle s'inscrit dans une logique de domination de celui qui sait sur celui qui ne sait pas. Toutes les garanties sont monétisées. "La traduction est un marché », «la langue est une marchandise » (Stahuljak op. cit.).

\section{Paris-Constantinople : l'École des jeunes de langue}

19 La création en 1669 par Colbert de l'École des jeunes de langue procède de la double nécessité de former des drogmans à la fois compétents et fiables pour les Échelles du Levant. Le texte de l'arrêté peut être consulté sur le site de l'Inalco ${ }^{1}$ :

Le Roy ayant reccu diverses plaintes des Marchands et négocians françois aux Eschelles de Levant, et particullièrement par ceux de Constantinople et Smirne, que les drogmans et interprettes, qui sont employés, abusent de la fonction de leur ministère et commectent divers abus qui ruinent et destruisent le Commerce, [...] ordonne sa Majesté que de trois en trois ans seront envoyéz auxdictes eschelles de Constantinople et Smirne, six jeunes garçons de l'aage de neuf et dix ans, qui voudront vollontairement y aller et iceux remis dans les couvent des Capucins desdits lieux de Constantinople et Smirne, pour y estre eslevéz et instruicts à la religion catholique, apostolique et romaine, et à la connoissance des langues en sorte qu'on puisse s'en servir avec le temps pour interpretter desdites langues [...]. 
Comme le suggère Marie-Hélène Clavères (2002), cette école est la première institution consacrée à l'apprentissage des langues modernes imaginée en France. Les débuts sont toutefois peu concluants. En 1700, on double le système par une formation au collège de Clermont tenu par les jésuites à Paris destinée à des groupes de jeunes "Arméniens ", nom collectif attribué à de jeunes Grecs, Syriens, Arméniens... Deux écoles désormais, l'une à Paris, l'autre à Constantinople. En 1721, on réorganise les programmes. Les élèves sont plus âgés, on instaure un système, plus efficace, d'alternance entre la formation en France et la formation chez les frères capucins de Constantinople (Dupont-Ferrier 1922, 1925 ; Hitzel 1996, 1997, 2013).

\section{Traducteurs et drogmans dans l'Empire ottoman, XIX siècle}

21 Au début du XIX ${ }^{e}$ siècle, plusieurs mesures sont prises visant à moderniser l'empire. Le sultan Mahmut II accorde aux musulmans la possibilité d'être nommés traducteurs ou drogmans, mesure impensable auparavant pour de multiples raisons, religieuses en particulier ${ }^{2}$. Est créé en 1821 au sein de la Sublime Porte le "Bureau de traduction" dont l'objectif est de former des interprètes et des traducteurs, et de façon plus générale d'enseigner les langues occidentales, notamment le français. Commence alors l'ère des drogmans issus de familles turques et musulmanes, et non plus seulement grecques, arméniennes, italiennes, françaises... Ce Bureau devient une véritable école de haut niveau qui va former en quelques années les nombreux drogmans dont le pays a besoin mais aussi des personnalités illustres de l'Empire, intellectuels, hommes d'État, diplomates, certains devenant de réels médiateurs entre l'Orient et l'Occident (Güven 2001 ; Timur Agildere 2007, 2009).

\section{Conclusion}

D'autres exemples pourraient, devraient compléter ce rapide panorama. Parmi d'autres: les écoles de cadets en Russie ou en Espagne; les écoles pilotées par les chambres de commerce et d'industrie françaises; les établissements d'enseignement technique de l'Alliance israélite universelle; les manuels destinés aux jeunes tirailleurs " sénégalais " conçus par l'armée française. S'agissant de publics dits spécialisés, les décisions visant l'apprentissage $\mathrm{du}$ français, ou d'autres langues, sont à peu près toujours liées à une conjonction d'ambitions politiques, économiques, scientifiques, voire expansionnistes et même parfois philanthropiques, affirmées, chaque institution ayant ses objectifs et chaque situation ses particularités.

\section{Les années 1960-1985}

Vers le milieu des années 1960, on assiste à un intérêt croissant pour l'enseignement du français à diverses catégories de publics spécialisés, mouvement qui va s'accentuer dans les années suivantes, notamment tout au long des années 70 et au début des années 80, à l'initiative ou sous la pression des instances institutionnelles, universitaires ou ministérielles concernées, françaises en particulier ${ }^{3}$. 


\section{Le français spécialisé, années 1960, début des années 1970} CREDIF (Centre de recherche et d'étude pour la diffusion du français) dans la réalisation de travaux lexicographiques portant sur les langues de spécialité dans la lignée du Français Fondamental premier et deuxième degrés, notamment un Vocabulaire Général d'Orientation Scientifique $e^{4}$ et des dictionnaires contextuels consacrés à la géologie et à la vie politique, le tout étant lié à l'idée d'une progression en trois temps de l'apprentissage, un niveau I consacré au français général, puis un niveau II visant l'acquisition d'une langue commune à différentes sciences, suivi d'un niveau de perfectionnement consacré à la langue de spécialité choisie. Parallèlement, est réalisé un manuel expérimental, Voix et Images médicales, sur le modèle de Voix et Images de France, destiné à des débutants. Au long des années 1960, le CREDIF co-organise, avec l'AUPELF ${ }^{5}$ notamment, quelques colloques, séminaires et stages de formation portant sur les langues de spécialité. Diverses initiatives sont portées par les instances officielles françaises, d'autres par les instances locales, voire les deux réunies. Quelques exemples parmi d'autres: création d'un Centre scientifique et technique français à Mexico (1961), travaux sur le français des mathématiques à Damas, formation d'ingénieurs iraniens de physique nucléaire à Grenoble (années 1970), publication du manuel Le français scientifique et technique de Masselin et al. (1971), accueil de jeunes bacheliers chinois dans des classes préparatoires aux grandes écoles scientifiques à Lyon vers les années 1975, formations plus ou moins confidentielles de militaires étrangers en France... Le français dit instrumental est enseigné dès la fin des années 60 dans des universités d'Amérique latine, avec pour objectif majeur l'accès à des textes spécialisés. La demande semble forte, l'offre encore trop limitée. Les outils pédagogiques en vigueur sont jugés inadaptés, trop lents, trop généraux.

\section{Pressions institutionnelles}

C'est dans ce contexte que le ministère des Affaires étrangères demande au CREDIF d'organiser des séminaires de réflexion dits de «français fonctionnel » visant à répondre aux demandes, à confronter les expériences et surtout à imaginer de nouvelles solutions. Une première rencontre exploratoire de deux semaines est organisée en 1974. Elle sera suivie de rencontres régulières d'une durée de trois puis quatre semaines qui se tiendront chaque été de 1975 à 1979 à Aix-en-Provence, parallèlement au stage d'été habituel du Centre. Chaque session rassemble une trentaine de spécialistes français et étrangers, soit déjà expérimentés en la matière, soit devant occuper des fonctions les y confrontant ${ }^{6}$. Au total, et à titre d'exemple, en 1979, sur 30 participants 23 pays sont représentés. D'autres formules suivront, dans le cadre des formations habituelles du CREDIF (stages d'été, stages longs, missions).

On assiste dès lors à une montée en puissance d'un secteur à part dans l'enseignement du français. Une innovation et non des moindres est l'entrée dans la description des postes à pourvoir pour l'étranger par le ministère des Affaires étrangères de la mention «français fonctionnel », avec la création d'une sorte d' "aristocratie » du domaine, les connaisseurs du fonctionnel, placés au sommet de la hiérarchie. Il s'agit en fait surtout d'assurer la survie d'un enseignement du français en perte de vitesse dans de nombreux pays via la conquête de nouveaux publics.

Documents pour l'histoire du français langue étrangère ou seconde, 66-67 | 2021 
27 Le CREDIF est également chargé par ce même ministère d'organiser en avril 1979 une première rencontre inter-universitaire portant sur le programme des boursiers dits prioritaires, programme d'envergure lancé en 1976 dans le cadre du VII ${ }^{\mathrm{e}}$ Plan par les services du Premier ministre avec pour objectif majeur de former de futurs partenaires et décideurs économiques dans des pays en voie d'industrialisation, en l'occurrence neuf pays en Asie, Proche et Moyen-Orient, Amérique latine (Iran, Irak, Égypte, Corée, Singapour, Indonésie, Brésil, Mexique, Venezuela) avec lesquels la France espère avoir à l'avenir des relations privilégiées, programmes menés dans quelques universités et grandes écoles, axés sur les sciences de l'ingénieur et de la gestion. Deux rencontres suivront en 1980 et 1981, à Lyon et à Toulouse. Un ouvrage collectif issu de ces rencontres et publié sous l'égide de l'AUPELF rend compte des différentes facettes du dispositif : sélection des candidats, mise à niveau linguistique et formation scientifique (AUPELF/CREDIF 1982). D'autres pays seront impliqués par des programmes du même type (Syrie, Thaïlande, Hong Kong, Nigeria, Chine, Éthiopie). Tous ces programmes ont certes des implications didactiques fortes et innovantes, mais personne n'ignore que les enjeux sont avant tout politiques, stratégiques et économiques, avec comme fer de lance la promotion active du français comme vecteur de l'exportation des innovations scientifiques et technologiques françaises. Pour avoir un aperçu des flux concernés, le nombre d'étudiants formés par le programme dit " prioritaire » a été d'environ $1600 \mathrm{en}$ six ans, chiffre donné au moment de la publication de l'ouvrage mentionné ci-dessus.

\section{Le français fonctionnel et ses avatars}

Un domaine est constitué. Il a ses objectifs, ses adeptes, tout comme ses détracteurs. Les publications se multiplient ${ }^{7}$. Des polémiques surgissent: formations bassement utilitaires, inféodées à des intérêts mercantiles et expansionnistes, dimension culturelle de la langue abandonnée, etc. Il continue malgré tout d'exister sous des appellations qui évoluent au fil des années. On passe de français scientifique et technique à langues de spécialité, puis à français fonctionnel (à l'initiative du ministère des Affaires étrangères) ou à enseignement fonctionnel du français ou encore à communication spécialisée, puis à enseignement sur objectifs spécifiques, expression calquée sur l'anglais, avec des variantes telles qu'objectifs universitaires, professionnels. On continue jusqu'à aujourd'hui à parler de français instrumental (en Amérique latine essentiellement). Plus généralement, on utilise de façon neutre enseignement à des publics spécialisés pour désigner l'ensemble des cas particuliers évoqués. Les différentes appellations, qui ont fait couler beaucoup d'encre, en particulier le terme de "fonctionnel », correspondent à des options didactiques plus ou moins dominantes à un moment donné, l'accent étant mis plutôt sur la langue et ses variétés, sur l'usage qui peut en être fait, sur les objectifs visés, sur les publics ${ }^{8}$.

\section{Les publics}

Pour mémoire, dans les années 1976-1977 le Conseil de l'Europe souligne une évidence, celle de la diversité des publics susceptibles d'apprendre une ou des langues étrangères. On n'est pas ou plus tenu d'enseigner la même chose à tout le monde et se développent des analyses de besoins langagiers, supposés différents (jusqu'à un certain point) pour chacune des catégories repérées. 
30 Et il est de fait qu'on assiste à une notable diversification des publics visés ou intéressés. On voit apparaître des demandes de formation provenant de scientifiques ou de techniciens de différents domaines et de différents niveaux: mathématiciens, géologues, agronomes, ingénieurs de l'aéronautique ou du nucléaire..., soit étudiants plus ou moins avancés, soit professionnels. Les domaines représentés se multiplient au fil des années: architectes, économistes, informaticiens, techniciens du pétrole, professionnels $\mathrm{du}$ tourisme et de l'hôtellerie, fonctionnaires des organisations internationales..., tout cela en fonction de l'état des échanges qui existent ou se développent entre tel pays et les pays francophones. Les objectifs liés aux perspectives économiques étant dominants, les sciences humaines et sociales sont moins bien représentées dans les divers dispositifs mis en œuvre, ou du moins, moins affichées. Historiens, géographes, archéologues... ne sont toutefois pas totalement exclus, dans des universités ou des centres culturels, par exemple. Quant aux migrants adultes, des études spécifiques leur sont consacrées, au CREDIF notamment.

\section{Les analyses de besoins, un passage obligé}

31 Face à une demande réelle ou supposée d'apprentissage émanant de ces publics jusquelà sinon oubliés du moins "malmenés" par les approches considérées comme universelles et donc inadéquates, on assiste à de nombreuses tentatives de redéfinition des besoins langagiers de chacune des populations visées. On voit à cette fin fleurir force schémas faisant apparaître les multiples composantes à prendre en compte, professionnelles, institutionnelles, académiques, avec en perspective une définition de plus en plus fine des objectifs à atteindre au cas par cas. On énumère les situations dans lesquelles l'apprenant aura (probablement) à terme à manier la langue, avec en cascade les caractéristiques détaillées des situations en question, lesquelles une fois bien déterminées, bien analysées, bien découpées, pourront faire l'objet d'un enseignementapprentissage spécifique. La démarche, logique en apparence, n'a toutefois pas manqué de susciter d'emblée des polémiques assez vives ${ }^{9}$, et même des réserves sérieuses y compris de la part de ses promoteurs ${ }^{10}$. La transformation mécanique des besoins supposés en objectifs à atteindre, définis a priori par les autorités éducatives - le terme recouvrant toutes sortes d'instances -, ne tient pas compte des motivations, capacités, intentions, réticences parfois ${ }^{11} \ldots$ variables dans la durée de surcroît, des apprenants potentiels ou réels. Quelques enquêtes menées sur ce point font largement état de la complexité des attitudes, penchants, attentes des uns et des autres, difficilement solubles dans des approches par trop rigides ou prédéterminées; elles montrent que bien souvent les objectifs et procédures considérés a priori comme utiles, efficaces, adéquats par les décideurs, peuvent se révéler inutiles, inefficaces, inadéquats ( $c f$. l'enquête réalisée auprès de thésards chinois par Peytregnet, Kahn, Besse 1983).

Par ailleurs, dans le cas de demandes à caractère strictement professionnel, l'influence des (futurs) employeurs peut se révéler dominante, visant à favoriser, imposer, des acquisitions étroitement utilitaires, sans perspective un tant soit peu valorisante pour les individus concernés. Dans le cas des boursiers scientifiques dits prioritaires, objets plus que sujets de tractations politico-économiques visant à fournir à des pays en voie de développement des cadres formés «à la française », plusieurs écueils et handicaps majeurs apparaissent assez vite: formations délivrées en France venant se greffer difficilement sur des formations antérieures et des habitudes antinomiques d'apprentissage ; à terme, contribution non prévue à une fuite possible des cerveaux 
vers des pays technologiquement avancés et davantage en phase avec la formation reçue; et de façon plus générale, problèmes structurels posés par les transferts de technologies avancées vers des pays économiquement moins avancés, remettant éventuellement en question le principe même de ce type de dispositifs (Zagefka 1982). Toutes ces objections ont généralement été prises en compte, elles ont permis dans la plupart des cas de revisiter la notion même d'analyse de besoins langagiers, voire de l'abandonner au profit de démarches mieux adaptées aux divers publics, avec, par exemple, des négociations régulières et renouvelées avec les apprenants concernant les objectifs et les pratiques (Richterich 1985). Elles ont surtout permis de replacer, de façon générale, l'apprenant au centre de l'apprentissage.

\section{Conclusion : supports de formation et aspects linguistiques}

33 Faute de manuels prêts à l'emploi, l'élaboration de documents de travail a souvent été une des préoccupations des enseignants et des concepteurs de programmes. C'est l'un des points centraux qui ont dû être traités durant les séminaires cités plus haut et au cours des innombrables formations qui ont suivi. La vogue des documents authentiques aidant, on cherche tous azimuts. On tente surtout de rationaliser les choix ${ }^{12}$. Deux logiques prévalent : l'une centrée sur le domaine de référence, valant surtout pour les professionnels en exercice, l'autre sur l'acquisition de savoir-faire techniques et de méthodes de travail qui intéressent davantage les étudiants. Concernant les professionnels, l'aide des experts est souvent nécessaire, et dans certains cas, à défaut de cours en binômes coûteux à réaliser, ont été mises en place des formes utiles de collaboration entre spécialistes et professeurs de français. Dans le cas des étudiants, les exigences académiques, notamment en termes d'évaluation, peuvent être déterminantes. Chaque cas de figure a donné lieu à pléthore d'analyses détaillées des situations plausibles d'usage de la langue, des objectifs visés et de l'échelonnement possible des activités d'apprentissage; le tout prenant en compte les exigences institutionnelles, les personnels disponibles, les rythmes retenus, la langue d'origine des apprenants, les habitudes d'apprentissage et d'enseignement des uns et des autres, leurs motivations, sans oublier l'influence des décideurs, les courants didactiques à la mode ou simplement « tendance » à un moment donné.

Quant aux aspects linguistiques, toutes les formes d'approches jugées utiles ou en vogue durant la période ont été mises à contribution, à des fins d'analyse des supports retenus mais plus encore à des fins d'organisation des enseignements: études lexicologiques, analyses de discours, énonciation, approches sociolinguistiques, traditions rhétoriques et culturelles, sans oublier la dimension purement grammaticale, morphosyntaxique, de la langue, force de frappe de base des enseignants et des concepteurs d'outils, dimension incontournable in fine de toute situation d'apprentissage de la langue. 


\section{BIBLIOGRAPHIE}

\section{Un manuel}

BERLAIMONT, Noël de (1613). Colloquia et dictionario cum octo linguarum [...] Colloques ou Dialogues, avec un Dictionaire en huit languages, Latin, Flamen, Francois, Alleman, Espagnol, ltalien, Anglois \& Portuguez [...] Flissingae, apud Martinum Abrahami f. vander Nolck commorantem Typographia. Anno 1613. En ligne:https://play.google.com/books/reader?id=pJaoOU0tu60C\&pg=GBS.PP6\&hl=fr

Articles parus dans Documents pour l'histoire du français langue étrangère ou seconde (DHFLES)

AUBERT, Françoise (1993). « Apprentissage des langues étrangères et préparation au voyage. À propos d'un manuel plurilingue attribué à Berlaimont ». DHFLES 11, 14-20.

CEPUKIENIENE, Stefa (1998). « Les débuts de l'enseignement du français en Lituanie ». DHFLES 21, 130-146.

CHADEFAUD, Catherine (2012). « Edme-François Jomard (1777-1862) et la mission égyptienne dans la première moitié du XIX ${ }^{\mathrm{e}}$ siècle ». DHFLES 49, 53-68.

CLAVÈRES, Marie-Hélène (2002). « L'enseignement de l'arabe au lycée de Marseille au XIX ${ }^{\mathrm{e}}$ siècle ». DHFLES 28, 177-202.

GOHARD-RADENKOVIC, Aline (1998). « Manuel de correspondance commerciale française ou le français à des fins professionnelles au début de ce siècle ». DHFLES 21, 147-165.

GRAZIANI, Jean-François (2012). « Les conséquences de la Première Guerre mondiale sur la politique culturelle et linguistique de la France au Japon (1917-1927) ». DHFLES 49, 167-182. GÜVEN, Hanife (2001). « Le rôle et la place du français dans le processus de modernisation de l'empire ottoman ». DHFLES 27, 73-82.

IBRAHIM, Amr Helmy (2007). « 1798-1976. Le français référentiaire des élites égyptiennes ». DHFLES 38-39, 131-147.

MERGER, Marie-France (2009). « Giuseppe Sessa et sa Dottrina popolare in quattro lingue (1891) ou... réussirai-je à écrire une lettre commerciale avec cet ouvrage ?». DHFLES 42, 215-230.

RIFFIER, Jean (2001). « Les œuvres françaises en Syrie à la veille de la Première Guerre mondiale : compétitions linguistiques et rivalités coloniales ». DHFLES 27, 83-96.

ROIG, Carmen (1996). « Le rôle du français dans le développement des sciences expérimentales ». DHFLES 18, 397-404.

TIMUR AGILDERE, Suna (2007). « Les 'élites' de la Sublime Porte ou les médiateurs francophones du Bureau de traduction (Tercüme Odasi) au XIX siècle ». DHFLES 38-39, 183-191.

\section{Du Moyen Âge au début du XX $X^{\mathrm{e}}$ siècle}

BALIVET, Michel (1997). « Avant les jeunes de langue : coup d'œil sur l'apprentissage des langues turques en monde chrétien, de Byzance à Guillaume Postel (VI ${ }^{\mathrm{e}}-\mathrm{XVI}^{\mathrm{e}}$ siècles) ». In Frédéric Hitzel (éd.). Istanbul et les langues orientales. Paris : L'Harmattan, 67-77.

BÉLY, Lucien (2017). «L'usage diplomatique de la langue française, instrument de la puissance ?». In Karène Sanchez-Summerer \& Willem Frijhoff (eds.). Linguistic and Cultural Foreign Policies of European States: 18th-20th Centuries. Amsterdam : Amsterdam University Press. 
BRIZAY, Bernard (2013). La France en Chine. Du XVII siècle à nos jours. Paris : Éditions Perrin, coll. Synthèses Historiques, 454-456.

CHARLET-MESDJIAN, Béatrice \& CHARLET, Jean-Louis (2011). « Une méthode Assimil pour apprendre le latin à l'époque humaniste : les Colloquia dérivés du Vocabulare de Noël de Berlaimont ». Rursus, 6. En ligne : https://journals.openedition.org/rursus/495

COLOMBO TIMELLI, Maria (1992). « Dictionnaires pour voyageurs, dictionnaires pour marchands, ou la polyglossie au quotidien aux XVI ${ }^{\mathrm{e}}$ et XVII ${ }^{\mathrm{e}}$ siècles ». Linguisticae Investigationes, XVI / 2, 395-420.

DELACROIX-BESNIER, Claudine (2012). « De l'usage des langues orientales dans les missions

catholiques (XIII ${ }^{\mathrm{e}}-\mathrm{XV}^{\mathrm{e}}$ siècle) ». In François Brizay (dir.). Les formes de l'échange. Communiquer, diffuser, informer, de l'Antiquité au XVIII siècle. Presses Universitaires de Rennes, coll. Histoire, 97-116. En ligne : https://books.openedition.org/pur/129642

DUPONT-FERRIER, Gustave (1922). « Les jeunes de langues ou “Arméniens” à Louis-le-Grand ». Revue des études arméniennes, III ${ }^{\mathrm{e}}$ année, tome II, 189-232. Paris : Imprimerie Nationale, Librairie Paul Geuthner. En ligne : https://ia800707.us.archive.org/27/items/revuedestudesa02soci/ revuedestudesa02soci.pdf

DUPONT-FERRIER, Gustave (1925). « Mémoire justificatif sur les jeunes des langues ou “Arméniens” à Louis-le-Grand 1669-1823 ». In La Vie quotidienne d'un collège parisien pendant plus de trois cent cinquante ans. Du collège de Clermont au lycée Louis-le-Grand (1563-1920). Paris : Éditions de Boccard, t. III, 347-448.

GLAISE ANNE, Frédérique (2005). L'évolution sanitaire et médicale de la concession française de Shanghai entre 1850 et 1950. Thèse soutenue à l'université Lumière Lyon 2. En ligne : http://theses.univlyon2.fr/documents/lyon2/2005/glaise_af\#p=121\&a=TH.6.3.

HERZSTEIN, Rafaël (2009). « Les pères jésuites et les Maronites du Mont Liban : l'Université SaintJoseph de Beyrouth ». Histoire et missions chrétiennes, vol. 9, n 1, 149-175. En ligne : https:// www.cairn.info/revue-histoire-monde-et-cultures-religieuses1-2009-1-page-149.htm HERZSTEIN, Rafaël (2010). « Une présence française en Méditerranée orientale : la fondation de l'Université Saint-Joseph de Beyrouth (1875-1914)». Matériaux pour l'histoire de notre temps, vol. 99, $n^{\circ} 3$, 2010, 4-11. En ligne : https://www.cairn.info/revue-materiaux-pour-l-histoire-de-notretemps-2010-3-page-4.htm

HITZEL, Frédéric (1996). « Les Jeunes de langue de Péra-lès-Constantinople ». Revue du Dix-Huitième Siècle 28, 57-70. En ligne : https://www.persee.fr/doc/dhs_0070-6760_1996_num1_2092

HITZEL, Frédéric (éd.) (1997). Istanbul et les langues orientales. Actes du colloque organisé par l'IFÉA et

l'INALCO à l'occasion du bicentenaire de l'École des Langues Orientales, Istanbul 29-31 mai 1995. Paris : L'Harmattan.

HITZEL, Frédéric (2013). « L'École des Jeunes de langue d'Istanbul. Un modèle d'apprentissage des langues orientales ». In Gilbert Buti, Michèle Janin-Thivos \& Olivier Raveux (éds.). Langues et langages du commerce en Méditerranée et en Europe à l'époque moderne. Aix-en-Provence : Presses de l'université de Provence, 23-31. En ligne : https://books.openedition.org/pup/14522

MINERVA, Nadia \& PELLANDRA, Carla (dir.) (1997). Insegnare il francese in Italia. Repertorio analitico di manuali pubblicati dal 1615 al 1860. CLUEB.

STAHULJAK, Zrinka (2018). Les fixeurs au Moyen Âge, cycle de 4 conférences données au Collège de France en juin 2018 dans le cadre du séminaire de Patrick Boucheron. En ligne : https:// www.college-de-france.fr/site/patrick-boucheron/guestlecturer-2018-06-01-16h00.htm 
TANAKA, Sadao (1983). Les débuts de l'étude du français au Japon. Tôkyô : Librairie-Editions France Tosho.

TIMUR AGILDERE, Suna (2009). «Les interprètes au carrefour des cultures ou les drogmans dans l'Empire ottoman (XVI ${ }^{\mathrm{e}}$-début du XX ${ }^{\mathrm{e}}$ siècle). Babel, 55 / 1, 1-19.

TOLAN, John (2009. L'Europe latine et le monde arabe au Moyen Âge: cultures en conflit et en convergence. Presses Universitaires de Rennes, coll. « Histoire ». Chap. XII : « Porter la Bonne Parole auprès de Babel : les problèmes linguistiques chez les missionnaires mendiants, XIII ${ }^{\mathrm{e}}$-XIV ${ }^{\mathrm{e}}$ siècles ", 183-195. En ligne : https://books.openedition.org/pur/136440?lang=fr

\section{Le « fonctionnel ", seconde partie du $\mathrm{XX}^{\mathrm{e}}$ siècle}

AUPELF/CREDIF [KAHN, Gisèle \& LADOUSSE, André (coord.)] (1982). Transferts de formation : la mise à niveau linguistique et scientifique de cadres étrangers. AUPELF : Bibliothèque nationale du Canada.

CREDIF (1972). Vocabulaire général d'orientation scientifique, V.G.O.S., part du lexique commun dans l'expression scientifique. Sous la direction d'André Phal, avec la collaboration de Lucette Beis. Paris : Didier.

MASSELIN, Jacques, DUCHAIGNE, Robert \& DELSol, Alain (1971). Le Français scientifique et technique : tronc commun, technologie, physique, chimie. Paris : Hatier, 2 vol.

PEYTREGNET, Raphaël, KAHN, Gisèle \& BESSE, Henri (1983). Enquête préalable à la faisabilité d'une méthode de français pour boursiers scientifiques chinois. Saint-Cloud : ENS de Saint-Cloud/CREDIF, multigr., $79 \mathrm{p}$.

RICHTERICH, René (1979). «L'antidéfinition des besoins langagiers comme pratique pédagogique ». Le français dans le monde 149, 54-58.

RICHTERICH, René (1985). Besoins langagiers et objectifs d'apprentissage. Paris : Hachette, coll. F.

ZAGEFKA, Polymnia (1982). « La formation scientifique et l'industrialisation des pays en développement ». In AUPELF/CREDIF, Transferts de formation : la mise à niveau linguistique et scientifique de cadres étrangers, 49-69.

\section{NOTES}

1. En ligne: https://docplayer.fr/7845358-Les-origines-de-l-inalco-l-ecole-des-jeunes-delangues-fevrier-2014.html

2. Ainsi que le note Hitzel (2013 : 24) : « Aux yeux des chrétiens, apprendre les langues de l'islam n'allait pas sans péril pour l'intégrité de leur foi et pour leur salut. De leur côté, il était inconcevable pour un musulman de s'initier aux langues des 'vils mécréants', de même que l'on considérait comme une sorte de profanation à laisser les mécréants apprendre leurs langues. » La remarque vaudrait pour d'autres lieux et d'autres langues.

3. Les choix politiques en matière de diffusion du français durant cette période sont largement documentés. On pourra se reporter à Daniel Coste (dir.) (1984). Aspects d'une politique de diffusion du français langue étrangère depuis 1945. Matériaux pour une histoire. Paris : Hatier. Également à Denis Lehmann (1993). Objectifs spécifiques en langue étrangère. Les programmes en question. Paris: Hachette, coll. F.

4. Pour plus de précisions: André Phal (1969). «La recherche au CREDIF : la part du lexique commun dans les vocabulaires scientifiques et techniques ", Langue française 2. Le lexique, 1969, 73-81. 
5. Association des universités partiellement ou entièrement de langue française, fondée en 1961, devenue Agence universitaire de la Francophonie (AUF) en 1989.

6. Compte rendu et analyse dans Gisèle Kahn \& Denis Lehmann, « La mise en œuvre des principes $\mathrm{du}$ projet 'langues vivantes' dans le séminaire sur l'enseignement fonctionnel du français (enseignement aux publics adultes spécialisés) organisé à l'Université de Provence, centre d'Aix (période 1975-1979) par le C.R.E.D.I.F., École Normale Supérieure de Saint-Cloud », in Hans-Jürgen Krumm (coord.) (1986). Recueil de programmes de formation continue des enseignants répondant aux principes du Projet «langues vivantes» du Conseil de la coopération culturelle. Strasbourg: Conseil de l'Europe, 79-102.

7. Parmi d'autres: Louis Porcher (coord.) (1976). Approches d'un français fonctionnel. Études de linguistique appliquée 23 ; puis Gérard Vigner (1980). Didactique fonctionnelle du français. Paris: Hachette, coll. F ; Simone Eurin Balmet \& Martine Henao de Legge (1992). Pratiques du français scientifique. L'enseignement du français à des fins de communication scientifique. Paris: Hachette / AUPELF ; approches synthétisées dans Denis Lehmann (1993), cf. supra.

8. L'histoire des différentes appellations a fait l'objet de nombreuses publications; un exemple parmi d'autres: Gisèle Holtzer (2004). «Du français fonctionnel au français sur objectifs spécifiques. Histoire des notions et des pratiques». Français sur objectifs spécifiques: de la langue aux métiers. Le français dans le monde. Recherches et applications, $\mathrm{n}^{\circ}$ spécial, 8-24.

9. Parmi d'autres Henri Besse \& Robert Galisson (1980). Polémique en didactique. Du renouveau en question. Paris : CLE International.

10. À titre d'exemple: «Il n'y a donc pas une, mais des analyses, définitions ou identifications de besoins qui ont chacune leur but leur sens, leurs instruments, et leurs pièges. » (Richterich 1979: 56).

11. Décrites avec humour par Martine Henao \& Gin Heuman (1982). «Réflexion sur une expérience d'enseignement du français langue étrangère ». (AUPELF/CREDIF : 133-160).

12. Par exemple Gisèle Kahn (1998). "Des lecteurs et des textes ». Point Commun, la revue du français des affaires et des professions, 3. Chambre de Commerce et d'Industrie de Paris, 12-14. En ligne : https://www.lefrancaisdesaffaires.fr/wp-content/uploads/2019/12/Point-Commun-03Janvier-1998.pdf

\section{RÉSUMÉS}

Marchands, militaires, médecins, savants, interprètes... autant de spécialistes qui au fil des siècles ont été conduits à apprendre le français à des fins professionnelles. L'histoire de la diffusion des langues dans diverses régions du monde, du français entre autres, offre de nombreux exemples de situations économiques, scientifiques, éducatives, et plus largement géopolitiques, qui ont vu la mise en place de formations jugées nécessaires au développement de tel ou tel secteur, avec, entre autres, financements ciblés, équipes de recherche, circulation d'experts, attribution de bourses d'études. Plus récemment, dans les années 1960-1985 en particulier, et pour des motifs et des finalités proches des précédents, on assiste à un regain d'intérêt pour l'enseignement du français dit fonctionnel ou sur objectifs spécifiques, sous-tendu par une politique volontariste en la matière.

Traders, soldiers, doctors, scientists, interpreters... all professionals who, over the centuries, were bound to learn French for work reasons. A history of the spread of languages, French 
among others, in various parts of the world, shows many instances of economic, scientific, educational and, more generally, geopolitical situations which witnessed the setting-up of training curricula deemed necessary for the development of the various sectors of the economy, involving among others, targeted funding, research teams, expert visits, grants. More recently, in particular during the 1960-1985 period, for similar reasons and aims, there is renewed interest for the teaching of so-called functional French, or French for special purposes, supported by a dedicated policy in the field.

\section{INDEX}

Keywords : second language learning, French as a second language, functional French, French for specific purposes, language needs, French language policy, training policies, languages and development

Mots-clés : apprentissage des langues, enseignement du FLE, français fonctionnel, français sur objectifs spécifiques, besoins langagiers, politique linguistique, politique éducative, langues et développement

\section{AUTEUR}

\section{GISÈLE KAHN}

ENS de Lyon

gisele.kahn@gmail.com 